\title{
La Inteligencia Competitiva en los procesos de internacionalización empresarial. Una guía de recursos de información para la empresa española y el periodismo económico
}

\author{
Antonio MuÑOZ-CAÑAVATE \\ Universidad de Extremadura \\ amunoz@alcazaba.unex.es \\ Rosario FERNÁNDEZ-FALERO \\ Universidad de Extremadura \\ rferfal@alcazaba.unex.es
}

Recibido: 15/06/2015

Aceptado: 20/10/2015

\begin{abstract}
RESUMEN
La información se ha convertido en un recurso imprescindible para el proceso de toma de decisiones en las empresas, y elemento fundamental en el proceso de Inteligencia Competitiva. La globalización económica y la libre circulación de mercancías, bien sea dentro de estructuras supranacionales o derivados de la firma de Tratados internacionales, han aumentado el número de competidores presentes en los mercados, pero también las oportunidades para acceder a otros mercados internacionales.

Este trabajo describe los recursos de información más importantes que puede necesitar una empresa en su acceso a los mercados internacionales, y que proceden tanto de organismos públicos como privados. Palabras clave: España, Inteligencia Competitiva, recursos de información, fuentes abiertas, comercio exterior, medios de comunicación.
\end{abstract}

Competitive Intelligence in business internationalization processes. A information resource guide for the Spanish company and business journalism

\begin{abstract}
Information has become an essential part of any decision making process in a company and also of competitive intelligence. Economic Globalization and free market circulation have increased the number of international competitors. This situation leads to a broaden access to other markets in the world. This work describes the informational private and public resources that a company may need in order to access to any international market.

Keywords: Spain, Competitive Intelligence, information resources, open data, international Trade, media.

\section{Referencia normalizada}

Muñoz-Cañavate, A. y Fernández-Falero, R. (2015). "La Inteligencia Competitiva en los procesos de internacionalización empresarial. Una guía de recursos de información para la empresa española y el periodismo económico”. Documentación de las Ciencias de la Información, Vol. 38: páginas. 39-65
\end{abstract}




\section{INTRODUCCIÓN}

Los procesos de globalización económica han alterado de manera visible los distintos actores que operan en los mercados. Así, una consecuencia de la globalización ha sido la alteración de las fronteras económicas. Las empresas ya no compiten en entornos locales. La libre circulación de personas y mercancías dentro de estructuras supranacionales como la Unión Europea, o las nuevas reglas para el comercio internacional derivadas de la Organización Mundial del Comercio, han provocado un aumento del número de competidores, tanto para una pequeña empresa como para una multinacional, pero también de oportunidades en los nuevos mercados. Según la Unión Europea sólo un 25\% de las pymes exporta a mercados exteriores, y un porcentaje mucho menor lo hace fuera del territorio de la UE.

La información adquiere, así, un papel más protagonista. A las tareas tradicionales de gestión de la información interna -consustanciales a las organizaciones- y de gestión de la información corporativa, se une una, cada vez más acuciante, necesidad de obtener información externa que permita reducir la incertidumbre del entorno. En el caso de los procesos de internacionalización empresarial los directivos y gestores requieren de información muy variada: la situación política o económica de los mercados de destino -implica un análisis del riesgo del país ${ }^{1}$ y de los mercados exteriores-, los competidores del lugar, los distribuidores de las mercancías o servicios, la legislación del territorio, las normas de fabricación y exigencias técnicas, $^{2}$ aranceles, etc. Pero también se necesita conocer las oportunidades existentes, desde las ayudas para acceder a los mercados exteriores a las licitaciones internacionales que permitan conseguir un contrato o venta, o el conocimiento de las herramientas necesarias para dar a conocer a la empresa exportadora en los mercados de destino, entre otros factores.

La literatura internacional ha reflejado este nuevo entramado de necesidades y oportunidades en las que la información juega un papel fundamental. Lawrence Welch ya señalaba en 1996 que las necesidades de información y de búsqueda de

\footnotetext{
${ }^{1}$ Los tres tipos de riesgo relativos a un país son: el riesgo soberano, que es el riesgo que tienen los acreedores de los Estados o de las entidades que están garantizados por ellos; el riesgo de transferencia que es el que tienen los acreedores extranjeros frente a los residentes en un país cuando el país no tiene solvencia para hacer frente a sus deudas; y los riesgos derivados de la actividad financiera internacional. En ocasiones también se habla del riesgo político debido a que los objetivos económicos no se alcancen como consecuencia de cambios y decisiones políticas de los gobiernos.

${ }^{2}$ Además de las normas de la International Standard Organization (ISO) y de sus correspondencias -si las hay- con las españolas de AENOR, existen organismos internacionales que establecen sus propios estándares para poder comercializar los productos. Como por ejemplo, es el caso de British Retail Consortium que reune a las principales asociaciones de minoristas en Reino Unido y que establece la norma BRC y que son utilizadas por muchas empresas, debido a sus estándares de calidad y seguridad alimentaria.
} 
fuentes de información para la internacionalización de las empresas supuso un cambio en las estrategias de investigación (Welch, 1996). Otros trabajos han analizado el intercambio de información entre las organizaciones locales y extranjeras en proyectos de internacionalización (Liang, 2011); o la identificación de los contenidos y las fuentes de información fundamentales para la internacionalización (Fletcher, 2012).

Esta nueva necesidad, la de información cualificada, provoca la emergencia de nuevas disciplinas en el último tercio del siglo XX. Aparecen: la Inteligencia Competitiva, cuya primera asociación -la SCIP- $^{3}$ se crea en Estados Unidos en 1986, la Inteligencia Económica, ${ }^{4}$ o múltiples variantes como la Inteligencia Territorial, o la propia Inteligencia Internacional que ya se mencionaba en los años sesenta (Beauvois, 1961), aunque desde el punto de vista formativo apenas haya tenido repercusión en los estudios de Información y Documentación, al menos en España (Muñoz-Cañavate, 2007).

Como canal de acceso a la información, Internet se ha convertido en el conducto de distribución de un complejo entramado de recursos de información que antes eran de más difícil acceso. Las empresas utilizan este canal para la captura de información en sus planes de acceso a los mercados exteriores (Nguyen, 2006; Nguyen \& Barrett, 2006) y aplican las técnicas y herramientas de la Inteligencia Competitiva (Pelsmacker et al., 2005).

Este trabajo presenta una selección de los organismos públicos y privados a través de fuentes abiertas, las denominadas Open Source. El objetivo de este trabajo es mostrar una relación estructurada de los organismos que proveen los recursos de información más importantes que pueden servir a la empresa española para conocer el entorno externo en el que se encuentra -directivos y gestores de información y documentación-. ${ }^{5}$ También debe servir a los profesionales de la Información y la Docu-

\footnotetext{
${ }^{3}$ Society of Competitive Intelligence Professionals, después transformada en Strategic and Competitive Intelligence Professionals.

${ }^{4}$ En España se ha comenzado a mencionar otro concepto, el de Inteligencia Económica, como consecuencia de la influencia francesa y de Alan Juillet encargado del departamento de Inteligencia Económica de Francia. Se entiende a la Inteligencia Económica como la disciplina encargada de la protección de los intereses económicos nacionales, lo que implica que desde las estructuras del Estado se proteja los intereses de las empresas de un país frente a amenazas externas. En los últimos años el Gobierno español ha anunciado la creación de un Sistema de Inteligencia Económica (SIE). En la Estrategia de Seguridad Nacional presentada 2013 se menciona la creación del SIE como uno de los mecanismos de coordinación que permiten el desarrollo de la seguridad económica y de sus herramientas de apoyo. Sin embargo en mayo de 2015 desde la Dirección General de Comercio Internacional e Inversiones se anunciaba el aplazamiento en la creación de dicho sistema.

${ }^{5}$ Aunque no se encuentra entre los objetivos de este trabajo. Es necesario mencionar el papel que juegan los buscadores como herramientas para la localización de información. Un usuario puede acceder directamente a una fuente de información o bien utilizar las búsquedas de Internet. Existe una opinión generalizada en pensar que Google es el buscador universal. Sin embargo depende de en qué países se trate lo es, o es desplazado por otros. Este aspecto es muy importante si lo que pretende una empresa es
} 
mentación a adentrarse en la gestión y asesoría de estos recursos informativos. Y finalmente a los profesionales de los medios de comunicación especializados en información económica, donde la información sobre mercados internacionales está cada vez más presente.

Incluye a: los organismos internacionales más representativos, los organismos públicos españolas -nacionales y regionales- más importantes dedicados a la ayuda a la internacionalización, los servicios de información de las agencias de seguros de crédito a la exportación, los organismos del ámbito del asociacionismo empresarial, las consultoras del sector, los medios de comunicación, y los instrumentos del sistema financiero.

\section{ORGANISMOS INTERNACIONALES}

Las organizaciones internacionales gestionan abundantes recursos informativos en sus respectivos ámbitos. Los organismos e instituciones internacionales conocen, desde una amplia perspectiva, la situación del mundo o de un conjunto de países, tanto general como sectorial. A través de sus estructuras controlan y generan información que puede ser muy útil para un proceso de toma de decisiones. Han desarrollado importantes sistemas de información -en muchos casos públicos y de acceso abiertoen Internet.

Para estructurar mejor la presentación de los recursos informativos de estas organizaciones aparecen por un lado los organismos internacionales universales, unos pueden encontrarse bajo los auspicios del sistema de Naciones Unidas y otros organismos no. Posteriormente se incluyen los organismos internacionales regionales, es decir aquellos que reúnen a países de un entorno geográfico determinado, aunque haciendo especial referencia a la Unión Europea. La información que distribuyen es muy variada y se compone principalmente de aquellos documentos que generan sus órganos de decisión (declaraciones, normativas,

dictámenes, comunicados) aunque también información de gabinetes de estudios y análisis, información estadística, etc. Conforman en muchos casos complejos sistemas de información con bases de datos de libre acceso.

lanzar información corporativa en determinados países en el marco de una estrategia de internacionalización. Desde hace muchos años, los buscadores se han convertido en plataformas publicitarias que han convertido en la base de sus negocios, y que por tanto permiten localizar a elementos del entorno inmediato, como competidores de un mercado, potenciales distribuidores, proveedores, etc. Así Baidú es en China y Yandex en Rusia los buscadores más importantes. Incluso si se quiere hacer una búsqueda especializada en Google los resultados serán diferentes si se busca en Google.com o en el dominio de cada país, donde el idioma específico determinará los resultados finales. Si tenemos en cuenta que los buscadores indexan la Internet abierta y que lo hacen con las fuentes de información más variada, habrá que definir la estrategia específica en cada buscador en función de las necesidades específicas. 


\subsection{Organismos del sistema de Naciones Unidas}

El sistema de Naciones Unidas incluye un conglomerado de organismos especializados que pueden clasificarse en:

a) Organismos especializados de cooperación preferentemente social, humanitaria, cultural y de turismo. Entre estos están: la Organización Internacional del Trabajo (OIT) <ilo.org>; la Organización Mundial de la Salud (OMS) < who.int>; la Organización de las Naciones Unidas para la Educación, la Ciencia y la Cultura (UNESCO) <unesco.org>; la Organización Mundial de la Propiedad Intelectual (OMPI) $<$ wipo.int>; y la Organización Mundial del Turismo (OMT) < unwto.org>.

b) Organismos especializados de cooperación en el campo de las comunicaciones. Son: la Unión Postal Universal (UPU) <upu.int>; la Unión Internacional de Telecomunicaciones (UIT) <itu.int>; la Organización de la Aviación Civil Internacional (OACI) <icao.int>; y la Organización Marítima Internacional (OMI) <imo.org> .

c) Organismos especializados de cooperación técnica en sentido estricto. Se encuentra la Organización Meteorológica Mundial (OMM) < wmo.int>.

d) Organismos especializados de cooperación económica y financiera. Se encuentran la Organización Internacional para la Agricultura y la Alimentación (FAO) $<$ fao.org>; el Fondo Internacional de Desarrollo Agrícola (FIDA) <ifad.org>; la Organización de Naciones Unidas para el Desarrollo Industrial (ONUDI) < unido.org>; y el Fondo Monetario Internacional (FMI) <imf.org>.

e) Finalmente el grupo del Banco Mundial ${ }^{6}<$ bancomundial.org $>$; la Agencia Internacional de la Energía Atómica <iaea.org>; y el Consejo Económico y Social $<$ un.org/es/ecosoc $>$.

Del sistema de Naciones Unidas existen algunas herramientas muy útiles que pueden ayudar a las empresas a los procesos de internacionalización: Doing Business del Banco Mundial, la División de Estadísticas de Naciones Unidas y la Organización de Naciones Unidas para el Desarrollo Industrial (ONUDI).

El Proyecto Doing Business <espanol.doingbusiness.org> tiene como finalidad elaborar y difundir una base de datos cuya objetivo es analizar, comprender y mejorar las normas que regulan la actividad empresarial, para ello recopila, analiza y compara las normas reguladoras de la actividad empresarial de las pequeñas y medianas empresas seleccionadas, a lo largo de su ciclo de vida.

La División de Estadísticas de Naciones Unidas < unstats.un.org/unsd/statcom/

\footnotetext{
${ }^{6}$ Incluye el Banco Internacional de Reconstrucción y Fomento (BIRF), el Centro Internacional de Arreglo de Diferencias Relativas a Inversiones (CIADI), la Asociación Internacional de Fomento (AIF), la Corporación Financiera Internacional (CFI) y el Organismo Multilateral de Garantía de Inversiones (MIGA).
} 
commission.htm> es el organismo de mayor rango que toma decisiones sobre las actividades estadísticas internacionales (establecimiento de estándares estadísticos, o desarrollo de conceptos y métodos), así como sobre su implementación a nivel nacional e internacional.

Finalmente, la ONUDI ha desarrollado varios instrumentos en base a programas formativos online orientados a la exportación. Ha puesto en marcha el programa "Consorcios de exportación" dedicado a fomentar las exportaciones de las pymes. Los consorcios de exportación son alianzas entre varias empresas para promover la exportación de los productos o servicios de las empresas que participan. De esta manera las empresas pueden mejorar sus posibilidades de exportación en tanto que comparten conocimientos y riesgos sobre el acceso a los mercados.

\subsection{Otros organismos internaciones fuera del sistema de Naciones Unidas}

Entre estos organismos destaca la Organización de Cooperación y Desarrollo Económico (OCDE) creada en 1961. ${ }^{7}$ Está formada por 34 países de cuatro continentes. El amplio repertorio de temas que trata ${ }^{8}$ le permite mantener una amplia estructura informativa en la que distribuye multitud de recursos informativos. Su sistema de información sobre comercio < www.oecd.org/trade> incluye:

- Services Trade Restrictiveness Index. Ayuda a identificar las políticas que restringen el tráfico comercial. Informa sobre los negociadores y responsables políticos que tienen la información y las herramientas necesarias para establecer acuerdos comerciales y abrir el comercio internacional de servicios.

- Trade in Value Added (TiVA). Iniciativa conjunta entre la OCDE y la Organización Mundial del Comercio. Mide el comercio en función del valor añadido mediante el análisis estadístico de las fuentes, tanto por industrias como por países, que suman valor a los productos y servicios que se exportan o importan. Por ejemplo un producto elaborado en un país puede requerir partes y/o materias primas procedentes de otros países. TiVA provee información sobre qué industrias y países intervienen en la cadena de producción.

- Trade Facilitation Indicators. Son indicadores que ayudan a los gobiernos a desarrollar y mejorar el comercio internacional, reduciendo costes y obteniendo mayores beneficios. Estos indicadores identifican áreas de acción y evalúan el impacto de la aplicación de posibles reformas.

\footnotetext{
${ }^{7}$ Tiene su antecedente en una organización similar creada en 1948, la Organización Europea de Cooperación Económica.

${ }^{8}$ Administración Pública, Agricultura y Alimentación, Asuntos Sociales, Migración y Salud, Ciencia y Tecnología, Comercio, Desarrollo, Desarrollo Urbano, Rural y Regional, Economía, Educación, Empleo, Energía, Energía Nuclear, Finanzas e Inversión, Impuestos, Industria y Servicios, Medio Ambiente, Transporte.
} 
- Export Credits Arrangement text. Como su nombre indica es un acuerdo internacional que proporciona apoyo oficial al uso de créditos para la exportación.

\subsection{Otros organismos internacionales regionales ${ }^{9}$}

Existen un número elevado de organismos internacionales de ámbito regional que normalmente giran en torno a un continente. ${ }^{10}$ Destacan -al margen de la Unión Europea que se trata en el siguiente apartado- la Asociación Europea de Libre Comercio <efta.int>, la Organización de Estados Americanos <oas.org>, la Liga de Estados Árabes < arableagueonline.org>, la Unión Africana <au.int>, y la Organización de Cooperación Económica Asia-Pacífico <apec.org>.

\subsection{La Unión Europea}

La compleja configuración político-administrativa de la Unión Europea ha creado a lo largo de los años una estructura de servicios y recursos de información destinada a ciudadanos y empresas. Estas estructuras en algunos casos han pervivido, adaptándose a los cambios tecnológicos, como los Centros de documentación europea, mientras que en otros casos se han reconfigurado, transformado o simplemente desaparecido.

2.4.1. Instrumentos de la Dirección General de Mercado Interior, Industria, Emprendimiento y Pymes, y de la Dirección General de Comercio

\subsubsection{Entreprise Europe Network}

La red Enterprise Europa Network (EEN) es una red distribuida entre las distintas regiones europeas. Es heredera de las desaparecidas redes Euro Info Centres e Innovation Relay Centre (IRC). Los Euro Info Centres ${ }^{11}$ (más conocidos mientras existían

\footnotetext{
${ }^{9}$ Díez de Velasco nos dice que dentro de esta categoría agrupamos aquellas organizaciones que están restringidas a un número limitado de Estados entre los que existen unas determinadas afinidades objetivas (contigüidad geográfica) y/o subjetivas (similitudes económicas, políticas, religiosas, etc.). DÍAZ DE VELASCO, Manuel. Las Organizaciones Internacionales. Madrid: Tecnos, 2008, p. 49-50.

${ }^{10}$ Puede consultarse la relación de organismos internacionales y sus fuentes de información más importantes en: MUÑOZ CAÑAVATE, Antonio. Recursos de información para la inteligencia competitiva. Una guía para la toma de decisiones. Gijón: TREA, 2012, capítulo 6.

${ }^{11}$ Fue en 1986 cuando la Comisión Europea presentó una comunicación al Consejo que analizaba la diversidad de informaciones relativas a las pequeñas y medianas empresas -lo que suponía un problema debido a su variedad- y que trataba de solucionarse a través de una red de ventanillas que estarían distribuidas por los países de la Comunidad Europea pero aprovechando tanto la experiencia que ya tenían algunos países en la distribución de información empresarial como utilizando estructuras ya existentes -instalaciones de servicios de información de mercados exteriores, cámaras de comercio o
} 
como Euroventanillas) y cuyo nacimiento se produjo en 1987 suministraban información a las empresas pero también recogían información de las mismas, mientras los IRC establecidos en 1995 tenían como finalidad estimular la transferencia de tecnología entre los Estados y promover los servicios de Innovación. Pero también es sucesora de dos redes dedicadas a poner en contacto a empresas de distintos países: Bc-Net (Business Cooperation Network) y BRE (Bureau de Rapprochement des Enterprises).

En 2008 se presentó EEN, cuyos servicios se pueden agrupar en tres bloques:

a) Oportunidades de cooperación empresarial. En este ámbito la red Identifica y difunde oportunidades comerciales entre las empresas; proporciona asesoramiento en diversos temas relacionados con la internacionalización de las actividades empresariales; organiza encuentros para fomentar los acuerdos de cooperación comercial entre las empresas participantes, y apoya en las negociaciones dirigidas a la consecución de acuerdos de cooperación empresarial.

b) Oportunidades de cooperación tecnológica. EEN identifica y difunde ofertas y demandas de tecnología entre empresas, universidades, centros tecnológicos y demás organizaciones dedicadas a la $\mathrm{I}+\mathrm{D}+\mathrm{i}$; ofrece a las empresas asesoramiento en temas directamente relacionados con la transferencia de tecnología como Derechos de Propiedad Industrial y financiación; organiza eventos de transferencia tecnológica para fomentar los acuerdos entre empresas y oferentes de tecnología; y apoya en las negociaciones dirigidas a la consecución de acuerdos de cooperación tecnológica.

c) Oportunidades para la participación en proyectos europeos. El sistema de EEN identifica y difunde búsquedas de socios para la formación de consorcios y la presentación de proyectos a convocatorias de programas europeos. Para apoyar este objetivo organiza jornadas de presentación de convocatorias para la participación en programas europeos de financiación y seminarios especializados de

consultoras privadas-. Se establecía que estas ventanillas tendrían: a) funciones de información comunitaria ante las empresas en lo referente a la reglamentación de mercado interior, las ayudas de la CEE, los préstamos, los programas de investigación y desarrollo comunitario y la política comercial exterior de la CEE; b) funciones de vigilancia sobre el mercado interior incluyendo la posibilidad de tramitar la presentación de ofertas para concursos públicos, quejas a propósito de eventuales trabas al mercado interior o notificaciones en materia de competencias; c) funciones de coordinación de iniciativas comunitarias y nacionales en materia de creación de empresas y redes tecnológicas; y d) funciones de información a partir de un centro suministrador conectado directamente con las ventanillas comunitarias y a las pymes por métodos diversos (no sólo informáticos). 
formación para la preparación de propuestas de participación en dichos programas europeos.

\subsubsection{Portal para internacionalización de las pymes}

Es un portal <webgate.ec.europa.eu/smeip> que incluye información diversa para la internacionalización de las pymes: normas y reglamentos, información sectorial, misiones comerciales, eventos de cooperación empresarial.

\subsubsection{Portal Tu Europa. Guía práctica para hacer negocios en Europa}

Es una guía <europa.eu/youreurope/business> disponible en los distintos idiomas de la UE que informa sobre aspectos básicos para crear una empresa y datos para acceder a los mercados internacionales como aduanas, las ventas en el extranjero, los requisitos de los productos, los contratos públicos, etc.

\subsubsection{Portal europeo para las PyMES}

Es un portal <ec.europa.eu/small-business> que reúne información sobre las pymes y una variedad de servicios de información consejos prácticos como, por ejemplo, las reglas del mercado de la UE, la forma de hacer negocios fuera de la UE, los socios comerciales, Innovación, investigación, derechos de autor, etc.

\subsubsection{Cluster Internationalisation}

Iniciativa que ayuda a la internacionalización de los clúster europeos a través de acuerdos sectoriales dentro y fuera de Europa.

\subsubsection{Base de datos de acceso a los mercados}

Herramienta <madb.europa.eu/madb/> de la Dirección General de Comercio con los siguientes apartados:

- Tariffs Database. Ofrece información sobre los derechos aduaneros y los impuestos internos efectivamente aplicados para cada producto para cada mercado de exportación.

- Procedures and Formalities. Ofrece una lista de los documentos aduaneros y otras formalidades exigidas para cada producto en los distintos países.

- Statistical Database. Datos estadísticos entre la Unión Europea y un país tercero.

- SPS: Sanitary and Phytosanitary Issues. Información sobre aspectos sanitarios y fitosanitarios.

- Rules of Origin. Origen de las mercancías que se exportan y calificación en virtud del sistema de preferencias arancelarias. 


\subsubsection{Centros Europeos de Empresas e Innovación (CEEI)}

Los Centros Europeos de Empresas e Innovación (CEEI) aparecen en la primera mitad de los años ochenta. En 1984 se abre el primero en Lieja (Bélgica). Como centros para el desarrollo regional, ofrecen ayuda a las iniciativas empresariales que tratan la innovación y entre los servicios de información que ofrecen se encuentra la búsqueda de mercados potenciales.

\subsubsection{La Oficina de Publicaciones de la UE}

Además de las redes y portales ya descritos la Unión Europea tiene disponibles en Internet sus publicaciones y bases de datos, que incluye importante información para la internacionalización empresarial, como legislación, jurisprudencia, licitaciones internacionales, propuestas de la Comisión, informes de los distintos organismos o encuestas europeas, entre otros recursos informativo-documentales.

La estructura informativa de la Oficina de Publicaciones de la UE, mantiene:

a) El sistema Eur-Lex <eur-lex.europa.eu>, incluye el Diario Oficial de la Unión Europea (DOUE), la legislación de la UE (tratados, directivas, reglamentos, decisiones, legislación consolidada, etc.), los textos preparatorios (propuestas legislativas, informes, libros verdes y blancos, etc.), la jurisprudencia de la UE (sentencias, autos, etc.), los acuerdos internacionales, y otros documentos públicos de organismos de la UE.

b) EU Bookshop <bookshop.europa.eu>;

c) El portal de datos abiertos de la UE < open-data.europa.eu>;

d) El Suplemento TED del DOUE dedicado a la contratación pública europea (ted.europa.eu);

e) EU Whoiswho <europa.eu/whoiswho/whoiswho.html>;

f) Cordis, Servicio de Información Comunitario sobre Investigación y Desarrollo $<$ cordis.europa.eu>;

g) N-Lex Portal común de acceso a la legislación nacional <eur-lex.europa.eu/nlex>.

\subsubsection{Eurostat}

La Oficina de Estadística de la Unión Europea reúne las estadísticas de los países de la Unión. Tiene como finalidad crear repertorios de datos estadísticos homogéneos entre todos los Estados. Las estadísticas se estructuran en nueve ámbitos temáticos: estadística general y regional, economía y finanzas, población y condiciones sociales, industria, comercio y servicios, agricultura y pesca, comercio exterior, transporte, medio ambiente y energía, y ciencia y tecnología. 


\subsubsection{Las ventanillas únicas}

Son una herramienta surgida por la Directiva 2006/123 relativa a los servicios en el mercado interior, pensada sobre todo para las pequeñas y medianas empresas, que tiene como finalidad principal facilitar el ejercicio de la libertad de establecimiento de los prestadores de servicios y la libre circulación de servicios.

Las ventanillas reunidas en la red EUGO <www.eugo.es> y con un portal en Internet para cada uno de los países de la Unión Europea, busca informar a través de un único punto de acceso sobre los trámites necesarios para acceder a las actividades de servicios y la solicitud de las autorizaciones necesarias en cada país de la UE. La Directiva establece qué información debe suministrarse a través de las ventanillas:

- Procedimientos y trámites necesarios para las actividades de servicios en cada territorio.

- Datos de las autoridades responsables de las actividades de servicios.

- Condiciones de acceso a los registros y bases de datos públicos relacionados con los servicios.

- Forma de recurrir ante los tribunales

- Datos de asociaciones a los que dirigirse para obtener ayuda.

\subsubsection{Otros instrumentos dentro de la UE}

En otros ámbitos se encuentran: el Eurobarómetro, servicio creado en 1973, para conocer la opinión pública de los Estados miembros, y la diversidad de agencias descentralizadas por todo el territorio de la Unión Europea. Entre ellas las dos agencias dedicadas a la protección de la propiedad industrial: la Oficina de Armonización del Mercado Interior (OHIM) y la Oficina Comunitaria de Variedades Vegetales (CPVO).

\section{ORGANISMOS ESPAÑOLES DEL ÁMBITO PÚBLICO}

En España se ha desarrollado toda una estructura de apoyo a la internacionalización de las empresas, que puede ser estudiada desde dos esferas: a) aquellos organismos, públicos o privados, cuyo ámbito de actuación es todo el país, y b) los organismos regionales, normalmente dependientes de los gobiernos regionales, que tienen como finalidad el apoyo a las empresas de su región.

En el caso de los organismos y entidades de ámbito nacional se describen el Instituto Español de Comercio Exterior, la Sociedad Estatal España “Expansión Exterior”, la Secretaría de Estado de Comercio, la red estatal de oficinas (Ofecoes), y la Compañía Española de Financiación del Desarrollo. 


\subsection{De Isabel II al Instituto Español de Comercio Exterior}

Las políticas de información para el apoyo a la internacionalización tienen en el Instituto Español de Comercio Exterior uno de sus ejes principales. Las políticas públicas para el apoyo al comercio exterior se remontan al siglo XIX. Durante el reinado de Isabel II se creó la Dirección de Comercio entre cuyos objetivos tenía el de facilitar a las compañías españolas el acceso a los mercados exteriores. También el Ministerio de Estado se convierte a finales del siglo XIX en uno de los apoyos más importantes para el fomento del comercio exterior, a través de dos organismos: el Centro de Información Comercial y la Junta de Comercio de Exportación. ${ }^{12}$ Posteriormente, en 1906 se establece en el Ministerio de Fomento el Centro Nacional de Informaciones Comerciales y Archivo de Sociedades Anónimas (CNICASA). ${ }^{13}$

Paloma Sendín (Sendín de Cáceres, 2005) nos dice que desde 1929 hasta 1982 las políticas de apoyo a la internacionalización se definen por la especialización. Surge el Banco Exterior de España y el Servicio de Agentes o Agregados Comerciales en el Extranjero. Será el Banco Exterior el que en 1952 establezca la Sociedad Anónima de Fomento de Comercio Exterior (Focoex) que busca impulsar el comercio exterior. Y en 1976 se crea el Centro de Documentación e Información Exterior (Cedin), el Boletín de Información Selectiva de la Información (BISE) y el Boletín de Información Comercial Española (BICE), entre otras publicaciones.

Las políticas públicas más recientes datan de 1982, año en el que se crea el Instituto Nacional de Fomento de la Exportación (INFE). En 1987 cambia su denominación a Instituto Español de Comercio Exterior (Real Decreto 1417/1987). El ICEX se transforma en 2011 en entidad pública empresarial (Real Decreto-ley 4/2011), con el objetivo de ganar eficacia y competitividad con tres objetivos específicos:

1. Promover la internacionalización de la empresa española y la mejora de su competitividad a través de las acciones de los Gobiernos en el marco de la política económica.

2. Facilitar especialmente a las pymes todas las fases de su proceso de internacionalización, con servicios de información, programas, financiación o asesoría.

\footnotetext{
12 a) El Centro de Información Comercial estuvo en funcionamiento entre 1898 y 1924. Sus funciones eran la de dar a conocer la oferta exportadora española y las demandas procedentes del exterior, apoyado en la red de embajadas españolas. También elaboraba estudios de mercado y publicaba el Boletín del Centro de Información Comercial. Posteriormente aumentará sus competencias con la puesta en marcha de un servicio de traducciones de pago, elaboración de catálogos de importadores y exportadores, información de aranceles, etc.

b) La Junta de Comercio de Exportación creada en 1899, entre sus competencias se encontraba la de proponer viajes de prospección mercantil.

${ }^{13}$ En este Ministerio ya existían dos órganos que terminarían integrándose en el CNICASA: el Consejo Superior de Producción y la Junta de Comercio Internacional. Y en 1910 el CNICASA es sustituido por el Centro de Comercio Exterior y Expansión Comercial.
} 
3. Y desarrollar actuaciones con otras instituciones públicas y privadas dedicadas a apoyar la internacionalización empresarial.

El Instituto Español de Comercio Exterior que había tenido un papel importante en el desarrollo de la industria de la información electrónica española, ${ }^{14}$ cambia su estrategia con la irrupción de Internet. La generalización de los productos de información electrónica, y el proceso de desintermediación de la consulta de los recursos de información, como consecuencia de la facilidad de acceso e interrogación de los productos en entorno web, hace que el ICEX estructure sus contenidos en su portal web <icex.es>. En la actualidad los recursos de información se distribuyen así:

- Programas y servicios de apoyo. Incluye, entre otras informaciones, datos de los planes y programas de apoyo a la internacionalización, asesoramiento en contratación internacional y licitaciones, y las guías de apoyo a la internacionalización. ${ }^{15}$

- Financiación para la internacionalización.

- Servicios a medida. Ofrecen consultoría internacional personalizados para más de 150 mercados y están gestionados a través de la red de Oficinas Económicas y Comerciales de España en el Exterior.

- Información de mercados. Incluye información sobre un número elevado de países del mundo. Información económica, financiera y política de cada país, relaciones bilaterales, estudios e informes sectoriales, trámites de acceso a los mercados y sectores de oportunidad, selección de noticias económicas, y enlaces a cámaras de comercio, directorios de empresas, estadísticas, informes y organismos financieros.

- Ayudas. Convocatorias para asociaciones y federaciones de exportadores o entidades que colaboren con el ICEX y para empresas.

\footnotetext{
${ }^{14}$ Editó junto a los organismos de comercio exterior de Francia, Italia y Portugal la base de datos CDExport; y fue responsable del conjunto de bases de datos conocido como SIBILA (Sistema Icex para la Búsqueda de Información. Lectura y Actualización) que incluía bases de datos bibliográficas, un directorio de empresas exportadoras (Oferes), la base de datos Estacom de estadísticas de comercio exterior, Syce con datos de las empresas exportadoras, y las oportunidades de comercio exterior canalizadas a través de las oficinas comerciales de España en el exterior (Bise).

${ }^{15}$ Las guías de apoyo a la internacionalización, con una para los servicios de la Administración General del Estado (incluye el ICEX), y 17 más que incluyen los servicios de apoyo al comercio exterior de cada región, recogen los instrumentos que ofrece la Administración central para impulsar su expansión en los mercados exteriores. Las ediciones regionales contienen los servicios que facilitan los gobiernos regionales para las empresas de su Comunidad Autónoma.
} 
- Información sobre ferias internacionales, misiones de inversores, encuentros empresariales y foros de cooperación empresarial.

- Oportunidades de negocio e inversión. Este apartado contiene demandas de importadores, distribuidores o usuarios finales extranjeros, oportunidades de inversión y privatizaciones, licitaciones de obras, suministros y servicios en países industrializados y oportunidades de negocio con financiación multilateral.

Un organismo diferenciado del ICEX y encargado de las políticas de fomento de las inversiones extranjeras en España ha sido Invest in Spain, nacido en 2005 como una Sociedad Estatal para la Promoción y Atracción de Inversiones Exteriores, ${ }^{16}$ pero integrado posteriormente en el ICEX como consecuencia del proceso de racionalización del sector empresarial público. De la misma forma el Centro de Estudios Económicos y Comerciales pasa también a depender orgánicamente del ICEX.

\subsection{Sociedad Estatal España, Expansión Exterior}

España Expansión Exterior <expansionexterior.es> es una empresa pública dedicada a asesorar a las empresas españolas en proyectos internacionales. Entre sus labores se encuentra la de buscar socios en los países donde se van a desarrollar los negocios y financiación, sobre todo a través de Bancos Multilaterales y Banca Privada internacional.

\subsection{Otros instrumentos de la Secretaría de Estado de Comercio}

La Secretaría de Estado de Comercio, además de los instrumentos ya señalados (ICEX, Expansión Exterior) estructura sus servicios de información a través de la Subdirección General de Inspección, Certificación y Asistencia Técnica del Comercio Exterior, ${ }^{17}$ la Red periférica de Direcciones Regionales y Territoriales de Comercio y las oficinas Económicas y Comerciales en el Exterior. El conjunto de políticas se

\footnotetext{
${ }^{16}$ Ministerio de la Presidencia. Resolución de 1 de abril de 2005, de la Subsecretaría, por la que se dispone la publicación del Acuerdo del Consejo de Ministros, de 25 de febrero de 2005, por el que se adoptan mandatos para poner en marcha medidas de impulso a la productividad (BOE, 2/4/2005).

${ }^{17}$ La Directiva 98/34/CE, establece el procedimiento de información en materia de normas y reglamentaciones técnicas con el objeto de garantizar la mayor transparencia posible y eliminar o reducir posibles obstáculos a la libre circulación de mercancías. En España la Subdirección General de Inspección, Certificación y Asistencia Técnica del Comercio Exterior ha establecido un canal directo de comunicación con las asociaciones de fabricantes y exportadores y les hace partícipes de los mecanismos de la Directiva, lo que permite conocer de una manera directa las opiniones de los sectores exportadores. Este canal de comunicación posibilita que se anticipen las medidas que permitan evitar posibles obstáculos al comercio.
} 
coordinan a través de los planes estratégicos de Internacionalización de la Economía Española. La Secretaría de Estado de Comercio desde su portal en Internet distribuye y/o agrega otros instrumentos informativos:

a) El portal web barrerascomerciales.es, ${ }^{18}$ puesto en marcha en 2009, es un instrumento que sirve como sistema de información sobre barreras en mercados exteriores. Lo hace a través de dos vías:

- Por un lado dispone de un cuestionario de detección de barreras para la resolución de problemas que encuentran las empresas en países no Miembros de la Unión Europea, en sus operaciones de exportación de bienes y servicios, inversión y aprovisionamiento de materias primas y productos energéticos.

- Y por otro a través de las fichas de barreras existentes, que sirven para informar sobre barreras al comercio y a la inversión que se pueden encontrar en mercados exteriores.

b) Base de datos de Legislación sobre Calidad Comercial de Productos Alimenticios y Seguridad de Productos Industriales objeto de Comercio Exterior <legisla.comercio.es>.

c) Base de datos de precios diarios y semanales de los productos hortofrutícolas en los principales mercados europeos y de EE.UU, y normas de calidad <iprecom.comercio.es>.

d) Base de datos Canal País. Incluye información de comercio exterior con una ficha por país.

e) Base de datos Datacomex < datacomex.comercio.es>. Estadísticas a medida sobre datos de Comercio Exterior. ${ }^{19}$

f) Además de las bases de datos anteriores, la Secretaría de Estado de Comercio estructura en su portal más información para la internacionalización, en ocasiones de

\footnotetext{
${ }^{18}$ La Secretaría de Estado de Comercio Exterior también solventa, a través de un Centro de Consultas, problemas ad hoc sobre las condiciones de acceso a mercados exteriores (aranceles, formalidades, requisitos...), importadores, distribuidores, agentes, o cualquier cuestión relacionada con la promoción comercial. Las consultas también pueden realizarse por correo electrónico o por teléfono.

${ }^{19}$ El Instituto Nacional de Estadística <ine.es > realiza y/o agrega de otros organismos diversas estadísticas e informes relativos al Sector Exterior: Encuesta de Comercio Internacional de Servicios y los índices de Comercio Exterior -producto de la encuesta anterior-, las Estadísticas de Comercio Exterior de Mercancías, el Informe Trimestral de Comercio Exterior español, los índices de Tendencia de la Competitividad. Los números 58 y 59 de Índice. Revista de Estadística y Sociedad que publica el INE están dedicados al sector exterior.
} 
con información que proveen las Oficinas Económicas y Comerciales de España en el Exterior:

- Política comercial. Incluye los acuerdos comerciales de la UE con países terceros, medidas arancelarias, medidas de defensa comercial, regímenes comerciales importación y exportación, política comercial multilateral, obstáculos al comercio con terceros países.

- Información sobre diversos acuerdos internacionales de comercio: Organización Mundial de Comercio, Conferencia de las Naciones Unidas para el comercio y el desarrollo y Organización para la Cooperación y el Desarrollo Económico (OCDE).

- Control de Calidad y Asistencia Técnica al Exportador. Información sobre la Inspección de calidad comercial. Asistencia analítica a través de la red de laboratorios de la Dirección General de Comercio e Inversiones.

- Control de Seguridad Industrial a la Importación.

- Informes de organismos internacionales (FMI, OCDE, Banco Mundial) sobre países.

- Informes sectoriales de productos industriales, material de defensa y de doble uso, agroalimentarios, de servicios.

- Instrumentos de Apoyo a la Internacionalización (ferias comerciales, asociaciones de exportadores).

\subsection{Las Oficinas Económicas y Comerciales de España en el Exterior}

Estas oficinas conforman una red de delegaciones en el exterior (Ofecoes) y tienen una íntima relación con el Instituto Español de Comercio Exterior y con las cámaras de comercio. La importancia de la red estriba en que su personal conoce el entorno local en el que se encuentra y permite una mejor ejecución de sus actividades como la ayuda en ferias y exposiciones, misiones comerciales, viajes para conocer los mercados, etc. Este personal, por otra parte, también confecciona una serie de productos informativos: estudios de mercado sectoriales, información sobre concursos y licitaciones internacionales, información sobre la oferta exportable española a importadores locales, e información sobre agrupaciones de exportadores. A través del sitio web $<$ oficinascomerciales.es> estructura la información de cada país.

Estas oficinas proveen buena parte de la información sobre países que suministra el portal web del Instituto Español de Comercio Exterior. Por otra parte, la Oficina de Información Diplomática, del Ministerio de Asuntos Exteriores, gestiona un portal en Internet donde incluye una ficha país con información de las relaciones entre España y cada país (datos básicos, economía, situación política, relaciones bilaterales). 


\subsection{Compañía Española de Financiación del Desarrollo (COFIDES)}

Otro de los organismos que pertenecen a esta estructura pública es COFIDES $<$ cofides.es>. Es una sociedad mercantil creada en 1988 dedicada a facilitar la financiación de proyectos de inversión en el exterior. Estos proyectos deben de facilitar la internacionalización de las empresas españolas y servir a la vez al desarrollo de los países que reciben estas inversiones. Los organismos europeos similares se reúnen en la Asociación Europea de Instituciones Financieras de Desarrollo <edfi.be>.

\subsection{Los organismos de apoyo a la internacionalización de ámbito regional ${ }^{20}$}

En el conjunto de las Comunidades Autónomas se han creado estructuras paralelas a las propias del Estado. Junto al Instituto Español de Comercio Exterior (tanto en sus servicios centrales como en sus delegaciones) los gobiernos regionales han creado, en su mayoría, organismos regionales que realizan funciones similares o idénticas al ICEX. La crisis económica internacional que tiene su origen en 2007 ha llevado a algunas regiones a reorientar sus políticas, bien por la supresión de oficinas exteriores, o a su fusión dentro de las instalaciones de la red de Oficinas Comerciales de España en el Exterior.

Para coordinar las políticas de internacionalización de las empresas entre las Administraciones central, regional y el sector privado se creó en mayo de 2006 el Consejo Interterritorial de Internacionalización con el objetivo de fomentar la colaboración entre el Gobierno central y los gobiernos regionales junto al sector privado en el proceso de internacionalización de empresas. Aunque las políticas de coordinación entre distintas Administraciones ya habían culminado años antes con el Plan de Iniciación a la Promoción Exterior (PIPE). ${ }^{21}$

Los organismos regionales de promoción exterior han establecido redes en diferentes países con distintos modelos. Los organismos pueden haber desarrollado estructuras propias en el exterior, o bien ofertar sus servicios a través de empresas de consultoría que se encuentran previamente ubicadas en cada país, o en algunos casos directamente a través de personal adscrito en las oficinas de la red estatal Ofecoes.

\footnotetext{
${ }^{20}$ Existe otro tipo de organismo regional dedicado a la captura de inversiones exteriores. Sólo algunas regiones disponen de un organismo específico (es el caso de Andalucía con Invest in Andalucia o Cataluña con Invest in Catalonia, como ejemplos), ya que en ocasiones la misma entidad dedicada a apoyar la internacionalización de las empresas de una región se dedica a buscar las inversiones exteriores. En algunas regiones estas políticas se desarrollan a través de los nodos locales o regionales de la Red Enterprise Europe Network.

${ }^{21}$ PIPE comenzó a funcionar en 1997, a través del acuerdo que alcanzaron el ICEX y el Consejo Superior de Cámaras de Comercio, junto a todas las Cámaras de Comercio y todas las Comunidades Autónomas, con el objetivo de conseguir pymes exportadoras.
} 
Los distintos organismos regionales realizan actividades similares: formalizan planes para la internacionalización de las empresas de sus regiones, analizan el potencial de exportación de una empresa, ayudan a la promoción exterior de las compañías a través de misiones directas (acciones de promoción en ferias en el extranjero) o misiones inversas (visitas a las regiones españolas de agentes extranjeros), ayudan a la participación en ferias internacionales, a la promoción en puntos de venta, la realización de estudios sectoriales, se organizan entrevistas individuales con contactos en el país de destino, y se organizan jornadas técnicas. En ocasiones se promueve la incorporación a la empresa de profesionales especializados para el desarrollo de un proyecto internacional, y la realización de acciones conjuntas entre empresas para acometer la llegada a algún mercado internacional.

Las labores de información y de consultoría se suministran a través de una estructura con dos soportes: a) las oficinas centrales de estos organismos en sus regiones, y b) la red de oficinas en el exterior, que realizan las labores de captación de oportunidades comerciales y de negocio. Esta doble estructura informativa proporciona datos e información sobre tarifas, aranceles, directorios de importadores y exportadores de los distintos mercados, información sectorial (estudios de mercado o notas menos elaboradas sobre diferentes sectores), sondeos prospectivos de mercado y localización de clientes potenciales, apoyo y asesoramiento en licitaciones internacionales, fichas de países (en algunos casos se detallan las relaciones entre el país y la región).

La siguiente tabla presenta los organismos regionales que dependen de cada Administración regional, aunque existen casos en los que dichos organismos regionales han creado plataformas en colaboración con otras entidades de la región como las cámaras de comercio, asociaciones empresariales y los nodos de la red europea Enterprise Europe Network.

Tabla 1. Organismos regionales de promoción exterior

\begin{tabular}{|l|l|l|}
\hline Región & Entidad regional & \\
\hline Andalucía & $\begin{array}{l}\text { Agencia Andaluza de } \\
\text { Promoción Exterior S.A. } \\
\text { (Extenda) }\end{array}$ & www.extenda.es \\
\hline Aragón & $\begin{array}{l}\text { Aragón Exterior } \\
\text { (AREX) }\end{array}$ & www.aragonexterior.es \\
\hline Asturias & $\begin{array}{l}\text { Sociedad de Promoción } \\
\text { Exterior Principado de } \\
\text { Asturias S.A. } \\
\text { (ASTUREX) }\end{array}$ & www.asturex.org \\
\hline Baleares & $\begin{array}{l}\text { Instituto de Innovación } \\
\text { Empresarial de las Islas }\end{array}$ & www.idi.es \\
\hline
\end{tabular}




\begin{tabular}{|c|c|c|}
\hline & Baleares (IDI) & \\
\hline Canarias & $\begin{array}{l}\text { Sociedad Canaria de } \\
\text { Fomento Económico } \\
\text { (PROEXCA) }\end{array}$ & www.proexca.es \\
\hline Cantabria & $\begin{array}{l}\text { Sociedad para el Desa- } \\
\text { rrollo Regional de Can- } \\
\text { tabria (SODERCAN) }\end{array}$ & www.gruposodercan.es \\
\hline $\begin{array}{l}\text { Castilla y } \\
\text { León }\end{array}$ & $\begin{array}{l}\text { Agencia de Innovación, } \\
\text { financiación e Interna- } \\
\text { cionalización Empresa- } \\
\text { rial }\end{array}$ & www.excal.es \\
\hline $\begin{array}{l}\text { Castilla la } \\
\text { Mancha }\end{array}$ & $\begin{array}{l}\text { Instituto de Promoción } \\
\text { Exterior (IPEX) }\end{array}$ & www.ipex.es \\
\hline Cataluña & ACC1Ó & www.acc10.cat \\
\hline Extremadura & Extremadura Avante & www.extremaduraavante.es \\
\hline Galicia & $\begin{array}{l}\text { Instituto Galego de } \\
\text { Promoción Económica } \\
\text { (IGAPE) }\end{array}$ & www.igape.es \\
\hline Murcia & $\begin{array}{l}\text { Instituto de Fomento } \\
\text { (INFO) }\end{array}$ & www.institutofomentomurcia.es \\
\hline Navarra & Sin entidad específica & \\
\hline País Vasco & $\begin{array}{l}\text { Agencia Vasca de Desa- } \\
\text { rrollo Empresarial } \\
\text { (SPRI) }\end{array}$ & www.spri.es \\
\hline La Rioja & $\begin{array}{l}\text { Agencia de Desarrollo } \\
\text { Económico de La Rioja } \\
\text { (ADER) }\end{array}$ & www.ader.es \\
\hline Valencia & $\begin{array}{l}\text { Instituto Valenciano de } \\
\text { la Exportación (IVEX) }\end{array}$ & www.ivex.es \\
\hline
\end{tabular}

\section{LOS SERVICIOS DE INFORMACIÓN DE LAS AGENCIAS DE SEGUROS DE CRÉDITO A LA EXPORTACIÓN}

Entre los instrumentos para la internacionalización existe el seguro de crédito a la exportación, que es un elemento destinado a asegurar las operaciones comerciales con el exterior a través de una cobertura de parte de los riesgos que supone dicha transacción. Los riesgos pueden ser de naturaleza comercial, política o por otras causas extraordinarias. Estas compañías asesoran a las empresas en las distintas fases de desarrollo comercial, y ayudan a las empresas a evaluar sus riesgos. En ocasiones han dispuesto herramientas gratuitas que informan de las dificultades de cada país. Varias 
de las compañías que operan en España están asociadas a la Unión de Berna, fundada en 1934 y que reúne a algunas de las más importantes compañías de seguros de crédito a la exportación en el mundo. ${ }^{22}$

La compañía más importante en España es la Compañía de Seguros de Crédito a la Exportación (CESCE). Está formada mayoritariamente por capital público y se encuentra dentro de un grupo en el que también está Informa-D\&B, y DBK, dedicada a la información sectorial.

Junto a CESCE se encuentran varias compañías privadas que además de la comercialización de los respectivos seguros han generado herramientas informativas de coyuntura.

Coface Ibérica <coface.es> que es la sucursal en España del grupo francés Coface se asentó en España como aseguradora de crédito en 1995. Entre sus instrumentos de información se encuentra el portal de riesgos <riesgopaiscoface.com> que incluye un mapa de evaluaciones de Riesgo País sobre 160 países. Mide el riesgo medio de las empresas de cada país, y para ello combina las previsiones económicas, financieras y políticas del país, la experiencia de pagos de Coface y el análisis del entorno empresarial.

Crédito y Caución <creditoycaucion.es> se fundó en 1929. Sus servicios de información se apoyan en la compañía Iberinform de informes comerciales y Cycomex, que es el departamento de comercio exterior dedicado al asesoramiento en operaciones comerciales en el extranjero. Pertenece al Grupo Atradius, que ofrece seguros de crédito y caución en 50 países. Tiene un servicio de análisis del entorno económico con indicadores de morosidad empresarial, crédito y solvencia, indicadores de mercado exterior e indicadores de coyuntura.

Euler Hermes <solunionseguros.es> pertenece al Grupo Allianz. Realiza informes con previsiones macroeconómicas y de insolvencias por países y sectores, e incluye los principales riesgos y oportunidades de las tendencias en auge. Publica semanalmente el Weekly Export Risk Outlook, un boletín de riesgo país en el que se analiza la situación del mundo por regiones (información sobre cambios en los ámbitos comercial y político de distintos países).

\section{LAS ASOCIACIONES EMPRESARIALES}

\subsection{Las Cámaras de comercio}

Desde el nacimiento de las Cámaras de Comercio, Industria y Navegación en 1886 estas instituciones han desarrollado instrumentos muy diversos de apoyo a las empresas. La última reforma a finales de 2014 reestructura el antiguo Consejo Superior de

\footnotetext{
${ }^{22}$ Forman parte de la Unión de Berna 49 compañías entre ellas CESCE, Atradius y Coface.
} 
Cámaras de Comercio de España por la nueva Cámara de Comercio de España, que representa a las 88 Cámaras de comercio españolas y a las 35 en el exterior. ${ }^{23}$

Los servicios para el comercio exterior de Cámaras de España se organizan en:

a) Servicios, que incluyen asesoría para la internacionalización, directorio de empresas subcontratistas que tiene como objetivo poner en contacto a las empresas con compradores internacionales, y bases de datos de importaciones y exportaciones en España. ${ }^{24}$

b) Trámites con: a) la expedición del certificado de origen que acredita el lugar de origen de las mercancías que condiciona los trámites arancelarios con la finalidad de satisfacer los requisitos aduaneros o comerciales; b) Tramitación del cuaderno

de admisión temporal de mercancías; ${ }^{25}$ legalización de documentos comerciales; y certificado de libre venta y consumo. ${ }^{26}$

c) Y ayudas. Se estructura en el Plan de expansión internacional para pymes ${ }^{27}$ y en los comités de cooperación empresarial que permite que empresas españolas puedan establecer contactos comerciales con diferentes países.

En el pasado los instrumentos de comercio exterior de las Cámaras de comercio compitieron con los creados por la Confederación Española de Organizaciones Empresariales, una relación que no estuvo exenta de tensiones. ${ }^{28}$

\footnotetext{
${ }^{23}$ Cámara de España se crea por la Ley 4/2014 en sustitución del Consejo Superior de Cámaras de Comercio. Representa y coordina todas las Cámaras de Comercio territoriales, ante instancias estatales e internacionales.

${ }^{24}$ Las bases de datos incluyen a las empresas españolas que exportan y/o importan bienes, con detalle de productos, países, años de actividad, información de contacto, volumen del comercio exterior de España -con detalle por productos, países, comunidad autónoma, provincia, precios, número de empresas-.

${ }^{25}$ El cuaderno de admisión temporal de mercancías permite que las mercancías puedan viajar fuera de las fronteras nacionales para que puedan ser expuestas en ferias y exposiciones, ser usadas para la realización de trabajos o servicios de tipo profesional o ser presentadas como muestras comerciales por representantes.

${ }^{26}$ Este certificado permite cumplir con uno de los requisitos para poder registrar productos o marcas en otros países. Es el caso de los productos que requieren autorizaciones sanitarias o de los importadores. El documento certifica que los productos son libre venta o comercialización en el país de procedencia.

${ }^{27}$ Implica cuatro módulos: Las elecciones iniciales de productos, clientes y mercados; acceso al mercado; plan de marketing; y plan de negocio.

${ }^{28}$ CEOE Internacional fue el instrumento de la Confederación Española de Organizaciones Empresariales (CEOE) para potenciar el comercio internacional entre las empresas españolas. Fue creado en diciembre de 2012 como sociedad anónima. Ofrecía servicios apoyándose en la red de contactos con las organizaciones similares a la CEOE en otros países. A finales de 2014 se decidió su disolución, marcando una estrategia diferente a la llevada hasta ese momento que en ocasiones había estado marcada por su enfrentamiento con las Cámaras de Comercio. La disolución de CEOE Internacional ha abierto la posibilidad para establecer un sistema de colaboración público-privado entre las organizaciones empresariales y las Cámaras de Comercio, para desarrollar proyectos e instrumentos para la internacionalización de la pyme española.
} 
Además de las Cámaras españolas en el interior, existe una red de 35 de Cámaras en el extranjero. Se encuentran en la red CAMACOES. ${ }^{29}$ Estas Cámaras ayudan a las empresas españolas a conocer los mercados del país en el que se encuentran (legislación, normativas, estudios de mercado, contactos con importadores, distribuidores...) y a difundir a las empresas españolas y sus productos en el exterior.

\subsection{La Asociación Española de Consultoras de Comercio Exterior (Acocex)}

Esta asociación nace en 2005 y reúne a consultores en comercio exterior. A través de los profesionales que están afiliados a la organización Acocex <acocex.com> ofrece diversos servicios de información y formación:

- Asesoramiento Internacional, que incluye: pre diagnóstico de las empresas para su internacionalización, análisis de mercados exteriores y elaboración de planes de negocio internacional.

- Consultoría Estratégica, que ofrece creación de estrategias internacionales, análisis de viabilidad y competitividad y acompañamiento a mercados internacionales.

- Inteligencia de mercado internacional: información sobre mercados concretos, consultas sobre partidas arancelarias, y legislación de comercio internacional, acceso a bases de datos aduaneras, listados de importadores y exportadores.

- Y gestión aduanera, financiera y jurídica, acciones de marketing, y asesoría en logística y licitaciones.

\subsection{El Club de Exportadores e Inversores Españoles}

El Club de Exportadores <clubexportadores.org> se funda en 1997 como una asociación empresarial de diversos sectores y tamaños pero con experiencia en los mercados internacionales. Según el Club de Exportadores la cifra conjunta de facturación de sus asociados equivale al 25\% del PIB español, y de la que el $40 \%$ corresponde a su negocio internacional, tiene unos 800.000 empleados y sus inversiones en el exterior constituyen el 50\% del total de la inversión española internacional.

\footnotetext{
${ }^{29}$ Aunque tienen sus orígenes en 1923 la estructura moderna de las Cámaras en el exterior se produce con el Real Decreto 786/1979 que es modificado posteriormente por el Real Decreto 1717/2004, por el que se establecen las normas reguladoras del Estatuto General de las Cámaras de Comercio Españolas oficialmente reconocidas en el extranjero.
} 


\subsection{Federación Internacional para la Promoción y Desarrollo de Negocios y Comercio Exterior (Infebex)}

Nace en 2014 a partir de Acocex con el objetivo de proveer a las organizaciones y empresas integradas asesoría y servicios para su implantación fuera de España. Infebex <infebex.es> ofrece servicios a las asociaciones integradas como arbitraje internacional, comunicación y prensa, organización de eventos internacionales, asistencia a ejecutivos desplazados con servicios de acogida y de acompañamiento en los primeros meses en el nuevo país de residencia, acompañamiento en destino en las visitas de negocios, interpretes... También ayuda en ferias internacionales.

\subsection{Cámara de Comercio Internacional (ICC)}

La Cámara de Comercio Internacional <iccwbo.org> es la organización empresarial que representa a nivel mundial los intereses empresariales. Se constituyó en París en 1919 donde tiene su sede. Está constituido por empresas que realizan comercio exterior, organizaciones empresariales, y Cámaras de Comercio. Tiene el rango de organismo consultivo ante la ONU y sus agencias especializadas. Además es ente consultivo de la Organización Mundial de Comercio, del Fondo Monetario Internacional, del Banco Mundial, OCDE, y Comisión Europea, entre otras instituciones.

En España existe un Comité español <iccspain.org> desde 1922 que tiene su secretaría en Barcelona. En su sitio web se encuentran las preguntas y respuestas a dudas presentadas sobre comercio exterior.

\section{CONSULTORAS, MEDIOS DE COMUNICACIÓN Y FERIAS}

Existen en España una serie de empresas de consultoría dedicadas al comercio internacional y de ayuda a las empresas para su salida a los mercados. En unos casos son consultoras generalistas de Inteligencia Competitiva, y en otros especializadas en comercio exterior. En ocasiones junto a los servicios adhoc que realizan soportan importantes recursos de información en sus portales web.

Entre las consultoras dedicadas al asesoramiento internacional, cuyos servicios incluyen investigación de empresas, proveedores e individuos, apoyo a la entrada de nuevos mercados, se encuentran: Anthelex International <anthelexinternational.es>, Fudamenta <fundamenta.es>, GET Grupo de Estudios Técnicos < $<$ getseguridad.com>, Global Chase <globalchase.eu>, Grupo TARIC <taric.es>, Grupo Gesterec $<$ grupogesterec.com $>$, H4dm $<\mathrm{h} 4 \mathrm{dm} . \mathrm{dom}>$, Hiperion Advisers $<$ hiperionadvisers.com $>$, Infocenter $<$ infocenter.es $>$, Infoline $<$ infoline.es $>$, Id Inteligencia <ideinteligencia.com>, Miniera $<$ miniera.es $>$, Pons Patentes y Marcas Internacional <ponspatentesymarcas.es>, Prospektiker <prospektiker.es>, Redfle- 
xion Consultores < redflexion.com>, SSC Corporate Intelligence Consulting <ssconsulting.es $>$, S21sec $<$ s21sec.com>, Triz XXI <triz.es $>$.

En España operan diversas consultoras internacionales sobre Inteligencia, como Kroll <es.kroll.com>, K2 Global <k2globalconsulting.com> o FTI Consulting <fticonsulting.com.es>. Y varias compañías de gestión del riesgo internacional: Marsh $<$ marsh.es $>$ que realiza estudios sobre los conflictos internacionales, mapas de riesgo político, siniestralidad del seguro de crédito, y análisis de mercados; AON <aon.com/spain>; y Baker Tilly España <bakertilly.es>. A nivel internacional pero sin sede en España destacamos a Maplecroft <maplecroft.com>.

La internacionalización y el comercio exterior también se apoyan en la industria de los medios de comunicación -tanto del sector público como del privado-, especializados en economía o generalistas, y en todas sus formas y formatos: prensa, radio, televisión, portales de internet... Los medios de comunicación absorben la información tanto del entorno externo inmediato -clientes, competidores, proveedores, distribuidores- como del entorno externo remoto -situación política, económica, social o tecnológica-, de manera que pueden presentar la realidad de una empresa competidora en un mercado internacional, describir la situación política y económica de un país y su estabilidad o inestabilidad para realizar comercio exterior, etc.

Junto a los medios tradicionales y sus réplicas en Internet se ha conformado una industria de la información especializada en comercio exterior. En este ámbito encontramos a Iberglobal <iberglobal.com> que ha desarrollado una serie de productos informativos a los que se accede por suscripción, aunque en algunos casos la información está abierta de manera gratuita. ${ }^{30}$ Es responsable de un portal específico sobre China, denominado Iberchina <iberchina.org> y de Spain Global <spainglobal.com> que está dirigido a profesionales españoles o de otras nacionalidades que se encuentran fuera de España y que tienen interés en disponer de información sobre la actividad internacional de organizaciones y empresas españolas en los países en los que viven.

En el ámbito de las publicaciones periódicas, la compañía Grupo Difusión Global De Negocios S.L. constituida en 2005 comienza la publicación de la revista especializada Empresa Exterior <empresaexterior.com>. La versión digital tiene una newsletter diaria. Y el Grupo Euroempresa edita desde 2001 la revista Moneda Única <monedaunica.net>. Por otra parte el ICEX edita la revista digital "El Exportador Revista de Internacionalización".

La generalización de Internet como herramienta de distribución de información ha llegado a casi todos los medios, muchos de los cuales han creado hemerotecas digita-

\footnotetext{
${ }^{30}$ Dispone de un área de formación online sobre internacionalización en colaboración con la Universidad Autónoma de Madrid, realiza el Índice de Valoración de la Inversión Española en el Exterior junto con El Club de Exportadores e Inversores Españoles.
} 
les. En este entorno surgen también las empresas de seguimiento de medios y las dedicadas a agregar contenidos de los medios que pueden crear enormes archivos de datos. Entre estos servicios destacan a nivel mundial ${ }^{31}$ Factiva $<$ global.factiva.com>, Lexis Nexis $<$ lexisnexis.com $>$ o Highbeam $<$ highbeam.com $>$.

Finalmente, las ferias comerciales son un medio eficaz tanto para conocer nuevos mercados, competidores, distribuidores, como para dar a conocer la oferta de una empresa a nuevos clientes. En España se encuentran InvExport (Salón de negocios internacionales) e IMEX - Impulso Exterior (feria de negocio internacional y comercio exterior).

\section{INDUSTRIA DE LA INFORMACIÓN: INFORMACIÓN COMERCIAL Y SECTORIAL}

Los contenidos enfocados a la internacionalización empresarial tienen en los informes comerciales y sectoriales dos productos básicos. La información comercial sobre competidores permite ver en un único documento una radiografía de una compañía. La variedad de datos desde su accionariado, cuentas anuales, marcas, e incluso rating -en algunos casos-, entre otros datos posibilitan conocer, al menos en una primera aproximación, a un posible competidor, distribuidor, proveedor en otros mercados. En España las principales compañías dedicadas a la venta de este tipo de productos pueden conseguir a través de sus redes internacionales informes comerciales de empresas de cualquier país del mundo. Destacan en este sector Informa D\&B $<$ informa.es>, Axesor <axesor.es> e Iberinform <iberinform.es >.

Por otra parte los informes sectoriales ofrecen una visión completa de la situación de un sector, además de la evolución que ha tenido en los últimos años. Algunas grandes entidades dedicadas a la elaboración y venta de informes sectoriales son: Euromonitor <euromonitor.com>, Datamonitor < datamonitor.com>, BDRC Continental <bdrc-continental.com>, y el agregador de informes de mercado Market Research Group <marketresearch.com>. Finalmente, The Economist Intelligence Unit <eiu.com> que pertenece al Grupo The Economist elabora informes sectoriales e informes de riesgo de casi 200 países, indicadores económicos, con análisis políticos y económicos.

\footnotetext{
${ }^{31}$ Para conocer con más detalle la situación de España, véase: MUÑOZ CAÑAVATE, Antonio. Recursos de información para la inteligencia competitiva. Una guía para la toma de decisiones. Gijón: TREA, 2012, p. 253-262.
} 


\section{LOS INSTRUMENTOS DEL SISTEMA FINANCIERO}

Las entidades del sistema financiero han generado herramientas que les han permitido luchar contra la morosidad a través del mantenimiento conjunto de ficheros de morosos y de solvencia, pero también han creado instrumentos y herramientas con la finalidad de ayudar a las empresas a exportar, a obtener información de los mercados, buscar socios, conocer información sobre las barreras arancelarias, etc. Las principales entidades financieras españolas han desarrollado estas estructuras en base a portales web, asesorías y consultarías ad hoc, y centros de formación a la exportación.

\section{CONCLUSIONES}

La generalización de Internet a mediados de los años noventa del siglo XX transforma de manera extraordinaria tanto la forma de acceder a la información, en otros tiempos de difícil captura, como la forma de distribuirla. Surgen, frente a los sistemas tradicionales, multitud de nuevas fuentes que utilizan la Red como el canal de distribución, de manera que los organismos ya existentes adaptan sus sistemas y métodos al nuevo entorno, a la vez que aparecen nuevos organismos directamente en el nuevo medio.

El mundo de gestión de información económica y empresarial no ha sido ajeno a estos cambios, donde cada vez más se requiere de fuentes de información fiables. Esta guía de recursos, a la vez que vale de herramienta para aquellas personas dedicadas al comercio internacional, necesitadas de herramientas informativas seguras, sirve a los profesionales de los medios de comunicación especializados en comercio internacional.

Este estudio ha realizado una aproximación a los organismos públicos y privados que generan las fuentes de información necesarias para la internacionalización, desde el conocimiento de un competidor existente en los mercados exteriores a las barreras que se puede encontrar una empresa en su salida exterior. Y demuestra el enorme potencial de este sector de la información.

\section{BIBLIOGRAFÍA}

BEAUVOIS, John J. International Intelligence for the International Enterprise. California Management Review, 1961, 3/2, pp. 39-46.

DÍAZ DE VELASCO, Manuel. Las Organizaciones Internacionales. Madrid: Tecnos, 2008.

FETCHER, Margaret; HARRIS, Simon. Knowledge acquisition for the internationalization of the smaller firm: Content and sources. International Business Review, 2012, 21/4, 631-647.

LIANG, Liang. The core-periphery structure of internationalization network in the tourism sector . Journal of China Tourism Research, 2011, 7/3, 282-293. 
MUÑOZ CAÑAVATE, Antonio. La enseñanza de la información para la empresa en la universidad española. Reflexiones para una orientación académica y profesional de la disciplina. Documentación de las Ciencias de la Información, 2007, 30, 253274.

MUÑOZ CAÑAVATE, Antonio. Recursos de información para la inteligencia competitiva. Una guía para la toma de decisiones. Gijón: TREA, 2012.

NGUYEN, Tho D. Factors affecting the utilization of the internet by internationalizing firms in transition markets: Evidence from Vietnam. Marketing Intelligence \& Planning, 2006, 25/4, 360-376.

NGUYEN, Tho D.; BARRETT, Nigel J. The adoption of the internet by export firms in transitional markets. Asia Pacific Journal of Marketing and Logistics, 2006, 18/1, 29-42.

PELSMACKER, Patrick De; MULLER, Marie-Luce; VIVIERS, Wilma; SAAYMAN, Andrea; CUYVERS, Ludo; JEGERS, Marc. Competitive intelligence practices of South African and Belgian exporters. Marketing Intelligence \& Planning, 2005, 23/6, 606-620.

SANTOS-ÁLVAREZ, María-Valle; GARCÍA-MERINO, María-Teresa. Información sobre internacionalización de empresas de Castilla-León. El profesional de la información, 2009, 18/4, 407-412.

SENDÍN DE CÁCERES, Paloma: «Evolución de la política de Promoción Comercial», Información Comercial Española ICE, 2005, 826, 209-221.

WELCH, Lawrence S. Information behavior and internationalization. International Journal of Technology Management, 1996, 11/1-2, 179-191 